九州大学学術情報リポジトリ

Kyushu University Institutional Repository

\title{
ON A STATISTICAL ANALYSIS OF HOMOGENEOUS RANDOM FIELDS ON A METRIC SPACE ACTED UPON BY A COMPACT METRIC GROUP
}

Nagai, Takeaki

Faculty of Science, Kagoshima University

https://doi.org/10.5109/13037

出版情報 : 統計数理研究. 13 (3/4), pp.65-92, 1969-03. Research Association of Statistical Sciences

バージョン：

権利関係 : 


\title{
ON A STATISTICAL ANALYSIS OF HOMOGENEOUS RANDOM FIELDS ON A METRIC SPACE ACTED UPON BY A COMPACT METRIC GROUP
}

\author{
By
}

\author{
Takeaki NAGAI*
}

(Received Jan. 5, 1969)

$\S 1$. Introduction and summary.

$\S 2$. Preliminaries.

§3. On the optimal linear interpolation of homogeneous random fields $I$.

$\S 4$. On the optimal linear interpolation of homogeneous random fields II.

\$. On the uniformly minimum variance unbiased linear estimates of mean value functions of homogeneous random fields.

$\S 6$. Appendix.

$\S 7$. Acknowledgment.

\section{$\S 1$. Introduction and summary.}

In this paper, we shall consider a problem on statistical analysis of second order homogeneous random fields $\{X(t), t \in T\}$ on a metric space $T$ acted upon by a compact metric group $G$ such that each $g \in G$ is a homeomorphism acting on $T$ and the mapping $(g, t) \rightarrow g t$ from $G \times T$ into $T$ is continuous.

The fields $\{X(t), t \in T\}$ are observable on a compact set $\Lambda \subset T$ invariant under every $g \in G$.

As preliminaries, we shall consider in $\S 2$ a measure $\mu$ on a compact metric space $\Lambda$ being a topological subspace of $T$ invariant under every $g \in G$ such that for every $g \in G$ and any Borel set $A$ of $A, \mu(g A)=\mu(A)$. By a measure on a metric space we always mean a non-negative countably additive set-function defined on the $\sigma$-field of Borel subsets of the metric space.

It is shown in $\S 2$ that there exists a Borel set $V \subset \Lambda$ such that (i) $G \cdot V=\Lambda$ and (ii) $G v_{1} \cap G v_{2}=\phi$ if $v_{1} \neq v_{2}\left(v_{1}, v_{2} \in V\right)$, (lemma 2.1) and that a mapping $\xi:(g, v) \rightarrow g v$ is a Borel isomorphism between $G \times V$ and $A$, (lemma 2.2). We shall call $V$ the Borel cross section of $A$ with respect to $G$ or simply the Borel cross section of $A$.

It is shown in theorem 2.1 that the induced measure $\mu^{*}=\mu \xi$ on $G \times V$ is decomposed into a direct product measure of the normalized Haar measure on $G$ and a measure $m$ on the Borel cross section $V$ in such a way that for any Borel set $A \times B \subset G \times V$,

$$
\mu^{*}(A \times B)=\nu(A) \cdot m(B)
$$

\footnotetext{
* Faculty of Science, Kagoshima University, Kagoshima.
} 
where $\nu$ is the normalized Haar measure on $G$ and $m$ is a measure on $V$. This result will be used in $\S 4$.

We shall consider in $\S 3$ the optimal linear estimator $\hat{Z}$ of a random variable $Z \in L_{2}(X(t), t \in T)$ such that $Z \notin L_{2}(X(t), t \in \Lambda)$ and $U(g) Z=Z$ for all $g \in G$ where for any $S \subset T L_{2}(X(t), t \in S)$ is a Hilbert space spanned by linear combinations of the field $\{X(t), t \in S\}$ under the scalar product $(U, W)$ defined by $(U, W)=E\{U \cdot \bar{W}\}$ and $\{U(g), g \in G\}$ are unitary operators on $L_{2}(X(t), t \in T)$ defined by writing $U(g) X(t)$ $=X(g t), t \in T$.

It is shown in theorem 3.1 that for any small $\varepsilon>0$ there exists a random variable $Y \in L_{2}(X(t), t \in V)$ such that

$$
E\left\{\left|\hat{Z}-\int_{G} U(g) d g \cdot Y\right|^{2}\right\}<\varepsilon
$$

where $V$ is a Borel cross section of $A$. The operator $\int_{G} U(g) d g$ is well-defined on $L_{2}(X(t), t \in \Lambda)$ and actually the projection operator onto the closed subspace $V_{1}$ consisting of all random variables invariant under every $U(g), g \in G$ in $L_{2}(X(t), t \in \Lambda)$.

The situations being the same as in $\S 3$, we shall proceed in $\S 4$ further in details to construct more practical approximating sequence $\left\{Y_{n}, n=1,2,3, \cdots\right\}$ for the optimal linear estimator $\hat{Z}$ of the random variable $Z$ invariant under every $U(g), g \in G$ such that

$$
\hat{Z}=\lim _{n \rightarrow \infty} \int_{G} U(g) d g \cdot Y_{n},
$$

where $Y_{n} \in L_{2}(X(t), t \in V), n=1,2,3, \cdots$, are given by the followings :

$$
Y_{n}=\int_{V} X(s) H_{n}(s) d m(s), \quad n=1,2,3, \cdots,
$$

where $\left\{H_{n}(s), s \in V, n=1,2,3, \cdots\right\}$ is a sequence of continuous bounded functions on $V$ which are determined by recursively and the measure $m$ is determined by a measure on $A$ invariant under every $g \in G$.

In $\S 5$ the metric space $T$ has the same properties as in the previous sections except for $T$ being compact and $T$ itself being invariant under every $g \in G$. We shall consider a second order homogeneous random field $\{X(t), t \in T\}$ with a mean value function $m(t)=E\{X(t)\}, t \in T$. We assume that the field is observable on $T$. Let $V$ be a Borel cross section of $T$ with respect to $G$, that is, $V$ is a Borel set of $T$ such that $G \cdot V=T$ and $G v_{1} \cap G v_{2}=\phi$ if $v_{1} \neq v_{2}\left(v_{1}, v_{2} \in V\right)$.

Then it is shown in theorem 5.3 that when the mean value function $m(t), t \in T$, is invariant under every $g \in G$, that is, $m(t)=m(g t)$, for all $g \in G$, and all $t \in T$, the uniformly minimum variance unbiased linear estimator $\widehat{m(t)}$ of the mean value function $m(t)$ at $t \in T$ is given by $\widehat{m(t)}=\int_{G} U(g) d g \cdot X(v)$, where for some $h \in G$ the point $t$ is written as $t=h v$.

As an appendix, we shall write in $\S 6$ several theorems which are refered to in our discussions. 


\section{$\S 2$. Preliminaries.}

In this section, we shall consider on decomposability of a measure $\mu$ on a compact set $A$ of a metric space $T$ acted upon by a compact metric group $G$.

Let $T$ be a metric space. Let $G$ be a compact metric group of homeomorphisms acting on $T$ such that the mapping $(g, t) \rightarrow g t$ from $G \times T$ into $T$ is continuous.

For any metric space $X$, we shall denote by $\mathfrak{B}_{X}$ the $\sigma$-field of Borel subsets of $X$, that is, $\mathfrak{B}_{X}$ is the smallest $\sigma$-algebra of subsets of $X$ containing all open sets.

Lemma 2.1. Let $A \subset T$ be a compact set invariant under every $g \leqq G$. Then, there exists a Borel set $V \subset A$ such that

$$
\begin{aligned}
& G \cdot V=A, \\
& G \cdot v_{1} \cap G \cdot v_{2}=\phi \quad \text { if } \quad v_{1} \neq v_{2} \quad\left(v_{1}, v_{2} \in V\right) .
\end{aligned}
$$

We shall call a Borel set $V$ satisfying (1) and (2) a Borel cross section of $A$ with respect to $G$ or simply a Borel cross section of $A$.

Proof. For any two points $t_{1}, t_{2}$ in $A$ we shall say that $t_{1} \sim t_{2}$ if there exists $g \in G$ such that $t_{1}=g t_{2}$. " $\sim$ " is an equivalence relation. Let $[t]$ denote the equivalence class containing $t \in A$. Let $M$ be the space of all such equivalence classes. The mapping $t \rightarrow[t]$ from $A$ into $M$ is continuous under the quotient topology and onto. Thus, the space $M$ is a continuous image of the compact set $A$ under the mapping and hence $M$ is a compact metric space.

Thus, by a theorem of Federer and Morse (see theorem 6.1 in $\S 6$ ) it follows that there exists a Borel set $V \subset A$ satisfying (1) and (2). The proof of lemma 2.1 is complete.

We shall show that the space $A$ may be identified with the product space $G \times V$.

Lemma 2.2. Suppose that for any $g \in G, g \neq e$, there is no fixed point in $A$.

Then, the mapping

$$
\xi:(g, v) \rightarrow g v
$$

is a Borel isomorphism between $G \times V$ and $\Lambda$.

Proof. From lemma 2.1 the mapping $\xi:(g, v) \rightarrow g v$ from $G \times V$ into $A$ is onto. Since there is no fixed point in $\Lambda$ for any $g \in G, g \neq e$, the mapping $\xi$ is one-one. From our assumption that the mapping $(g, t) \rightarrow g t$ from $G \times T$ into $T$ is continuous, it is clear that the mapping $\xi$ is continuous. In particular, $\xi$ is measurable.

Hence, by a theorem of Kuratowski (see theorem 6.2 in $\S 6$ ) the inverse mapping $\xi^{-1}$ is also measurable. Thus, we have proved lemma 2.2 .

Remark. Lemma 2.2 may be proved alternatively by making use of a theorem 6.3 in $\S 6$ in the virtue of the space $A$ being compact.

Let $\mu$ be a measure on $\Lambda$ invariant under every $g \in G$, that is, for each $g \in G$, $\mu(g A)=\mu(A), A \in \mathfrak{B}_{V}$.

Without loss of generality, we assume that $\mu(A)=1$, in other words, $\mu$ is a probability measure on $\Lambda$.

Let $\mu^{*}$ be the induced measure on $G \times V$ by the Borel isomorphism $\xi$ from the measure $\mu$ on $\Lambda$. 
Then, we have the following:

Theorem 2.1. The measure $\mu^{*}$ on $G \times V$ is decomposed into a direct product measure in such a way that for any Borel set $A \times B \subset G \times V$,

$$
\mu^{*}(A \times B) \equiv \mu(\xi(A \times B))=\nu(A) \cdot m(B),
$$

where $\nu$ is the normalized Haar measure on $G$ and $m$ a probability measure on the Borel cross section $V$ of $A$.

Proof. Both $G \times V$ and $G$ are compact metric spaces and hence these are complete separable metric spaces. Therefore, these are automatically separable standard Borel spaces (see [10], page 133).

Let us consider the mapping $\pi:(g, v) \rightarrow g$ from $G \times V$ onto $G$. Then it is clear that $\pi$ is measurable since the mapping $\pi$ is a projection from the product space $G \times V$ onto its coordinate space $G$.

Thus, it follows that there exists a regular conditional probability distribution of $\mu^{*}$ given $\pi$, which we shall denote by $\tilde{m}_{g}(A \times B), g \in G, A \times B \in \mathfrak{B}_{G \times v}$.

This satisfies the following conditions:

(i) For each $g \in G, \tilde{m}_{g}(\cdot)$ is a probability measure on $G \times V$.

(ii) For each Borel set $A \times B \subset G \times V$, the mapping $g \rightarrow \tilde{m}_{g}(A \times B)$ is $\mathfrak{B}_{G}$-measurable.

(iii) For any Borel set $A \times B \subset G \times V$,

$$
\mu^{*}(A \times B)=\int_{G} \tilde{m}_{g}(A \times B) d \nu(g),
$$

where $\nu$ is a probability measure on $G$ such that $\nu(E)=\mu^{*}\left(\pi^{-1}(E)\right)=\mu^{*}(E \times V), E \in \mathfrak{B}_{G}$. (See [10], page 146).

In particular, we have for any $E \in \mathfrak{B}_{G}$,

$$
\begin{aligned}
\mu^{*}\left(A \times B \cap \pi^{-1}(E)\right) & \equiv \mu^{*}((A \cap E) \times B) \\
& =\int_{E} \tilde{m}_{g}(A \times B) d \nu(g), \quad A \times B \in \mathfrak{B}_{G \times V} .
\end{aligned}
$$

Let us write

$$
m_{g}(B)=\tilde{m}_{g}(G \times B), \quad B \in \mathfrak{B}_{V} .
$$

Then we have by letting $A=G$ in (4),

$$
\mu^{*}(E \times B)=\int_{E} m_{g}(B) d \nu(g),
$$

for any $E \in \mathfrak{B}_{G}$ and any $B \in \mathfrak{B}_{V}$, where $m_{g}(\cdot)$ is a probability measure on $V$.

Since for each $g \in G$ and any Borel sets $A$ and $B$ of $G$ and $V$ respectively, $\mu^{*}(g A \times B)=\mu(\xi(g A \times B))=\mu(\xi(A \times B))=\mu^{*}(A \times B)$, it follows by letting particularly $B=V$ in (5) that for any $A \in \mathfrak{B}_{G}$,

$$
\nu(g A)=\mu^{*}(g A \times V)=\mu^{*}(A \times V)=\nu(A) .
$$

This equality and the uniqueness of the invariant measure on $G$ imply that the measure $\nu$ is the normalized Haar measure on G.

Thus, we may write

$$
\mu^{*}(A \times B)=\int_{\boldsymbol{A}} m(B) d g, \quad A \times B \in \mathfrak{B}_{G \times V} .
$$


Hence we have for any $A \in \mathfrak{B}_{G}, B \in \mathfrak{B}_{V}$ and $h \in G$,

$$
\begin{aligned}
\mu^{*}(A \times B) & =\int_{A} m_{g}(B) d g \\
& =\mu^{*}(h A \times B) \\
& =\int_{h A} m_{g}(B) d g \\
& =\int_{A} m_{h^{-1} \bullet g}(B) d g .
\end{aligned}
$$

This implies that for any $B \in \mathbb{B}_{V}$ and any $g, h \in G$,

$$
m_{g}(B)=m_{h}(B) \text {, }
$$

in other words, $m_{g}(\cdot)$ is a probability measure on $V$ independent of $g: G$.

Let us write

$$
m(B)=m_{g}(B), \quad B \in \mathfrak{B}_{V} .
$$

Then we have for any Borel set $A \times B \subset G \times V$,

$$
\begin{aligned}
\mu^{*}(A \times B) & =\int_{A} m(B) d g \\
& =\nu(A) \cdot m(B),
\end{aligned}
$$

where $\nu$ is the normalized Haar measure on $G$ and $m$ a probability measure on $V$. Thus, we have proved theorem 2.1 .

Example 2.1. Let $T=R_{2}=\{(x, y) \mid-\infty<x<\infty,-\infty<y<\infty\}$. Let $G=S O(2)$, the group consisting of all rotations on $T$ around the origin $(0,0)$. Let us consider a closed disk $A$ with a finite radius $r$, that is, $\Lambda=\left\{(x, y) \in R_{2} \mid 0 \leqq x^{2}+y^{2} \leqq r^{2}\right\}$.

It is obvious that as a Borel cross section of $A$, we may take a set $V=\{(x, 0)$ $10 \leqq x \leqq r\}$.

Since $S O(2)$ is isomorphic to the torus group $G_{0}=\{\theta \mid 0 \leqq \theta<2 \pi\}$ with the group operation $\theta_{1}+\theta_{2}=\theta_{3}$ where $\theta_{1}+\theta_{2} \equiv \theta_{3} \bmod 2 \pi, 0 \leqq \theta_{3}<2 \pi$, we may identify the space $A$ with the product space $V \times G_{0}$.

Let $\mu$ be a probability measure on $A$ such that for any $A \in \mathfrak{B}_{A}, \mu(A)$ is proportional to the "area" of the set $A$, in other words, $\mu$ is a uniform distribution on $A$.

Then $\mu$ is invariant under every $g \in S O(2)$ and it is written as follows:

For any Borel set $A \subset G_{0}$ and $B \subset V$,

$$
\int_{B \times A} d \mu(x, \theta)=\int_{B} \frac{2}{r^{2}} x d x \cdot \int_{A} \frac{1}{2 \pi} d \theta
$$

Example 2.2. Let $L_{3}=\left\{(t, x, y, z) \mid t^{2}-\left(x^{2}+y^{2}+z^{2}\right)=c^{2}\right\}$ where $t, x, y, z$ and $c$ are real numbers. Let $G$ be a subgroup of the proper Lorentz group of order (3.1) such that its elements are of the following form:

$$
g=\left(\begin{array}{ll}
1 & 0 \\
0^{\prime} & h
\end{array}\right), \quad h \in S O(3),
$$

where 0 denotes a zero vector $(0,0,0)$ and $0^{\prime}$ its transposed one.

Let $A=\left\{(t, x, y, z) \in L_{3} \mid 0 \leqq x^{2}+y^{2}+z^{2} \leqq \alpha^{2}\right\}$. where $\alpha$ is a finite real number. 
Since $A$ is invariant under every $g \in G$, we may take a set

$$
V=\left\{\left(\sqrt{c^{2}+u^{2}}, u, 0,0\right) \mid 0 \leqq u \leqq \alpha\right\} \subset A
$$

as a Borel cross section of $\Lambda$.

Let $\mu$ be a probability measure on $\Lambda$ such that for any $C \in \mathfrak{B}_{A}, \mu(C)$ is proportional to the "volume" of $C$, in other words, $\mu$ is uniformly distributed on $\Lambda$. Then $\mu$ is invariant under every $g \in G$. Thus, the measure $\mu$ is decomposed into a direct product measure as follows:

$$
\begin{aligned}
\int_{C} d \mu(x, y, z) & =\int_{B \times\left(A_{1} \times A_{2}\right)} d \mu(u, \theta, \varphi) \\
& =\int_{B} \frac{\beta u^{2}}{\sqrt{c^{2}+u^{2}}} d u \cdot \frac{1}{4 \pi} \int_{A_{1} \times \lambda_{2}} \sin \theta d \theta d \varphi
\end{aligned}
$$

where $C$ is a set of all points $(t, x, y, z)$ in $A$ such that

$$
\begin{aligned}
& x=u \cdot \sin \theta \cdot \cos \varphi, \\
& y=u \cdot \sin \theta \cdot \sin \varphi, \\
& z=u \cdot \cos \theta, \\
& t=\sqrt{c^{2}+u^{2}},
\end{aligned}
$$

$(\theta, \varphi) \in A_{1} \times A_{2} \in \mathfrak{B}_{G}$ and $u \in B \in \mathfrak{B}_{V}\left(u\right.$ is identified with a point $\left(\sqrt{c^{2}+u^{2}}, u, 0,0\right)$ in $\left.V\right)$ and

$$
\beta=2 \cdot\left[\alpha \cdot \sqrt{\alpha^{2}+c^{2}}+c^{2} \cdot \log \left(|c|-\left|\alpha+\sqrt{\alpha^{2}+c^{2}}\right|\right)\right]^{-1} .
$$

\section{§3. On the optimal linear interpolation of homogeneous random fields $I$.}

\subsection{Summary.}

In this section we shall consider an interpolation problem of a homogeneous random field $\{X(t), t \in T\}$ on a metric space $T$ acted upon by a compact metric group $G$ of homeomorphisms acting on $T$ such that the mapping $(g, t) \rightarrow g t$ from $G \times T$ into $T$ is continuous.

The field $\{X(t), t \in T\}$ is observable on a compact set $A \subset T$ invariant under every $g \in G$.

Let $Z \in L_{2}(X(t), t \in T)$ be a random variable invariant under all unitary operators $U(g), g \in G$ defined on $L_{2}(X(t), t \in T)$ by writing $U(g) X(t)=X(g t), t \in T$. Let $\hat{Z}$ $\in L_{2}(X(t), t \in \Lambda)$ be the best linear estimator of $Z$ on the basis of the realization of $X(t)$ on $\Lambda$.

Let $V$ be a subset of $A$ such that $A=G \cdot V$ and $G \cdot v_{1} \cap G \cdot v_{2}=\phi$ if $v_{1} \neq v_{2}\left(v_{1}, v_{2} \in V\right)$.

Then it will be shown the following result:

For each $\varepsilon>0$, there exists a random variable $Y \in L_{2}(X(t), t \in V)$ such that $E\left\{|\hat{Z}-A Y|^{2}\right\}<\varepsilon$ where $A=\int_{G} U(g) d g$ whose restriction to $L_{2}(X(t), t \in A)$ is a projection operator onto the maximal closed subspace $V_{1}$ invariant under all $U(g), g \in G$, that is, $V_{1}=\{u \mid U(g) u=u$, for all $g \in G\} \subset L_{2}(X(t), t \in T)$.

\subsection{An interpolation problem of homogeneous random fields.}


Let $T$ be a metric space. Let $G$ be a compact metric group of homeomorphisms acting on $T$ such that the mapping $(g, t) \rightarrow g t$ from $G \times T$ into $T$ is continuous.

Let $\{X(t), t \in T\}$ be a complex-valued homogeneous random field satisfying the conditions :

$$
\begin{array}{ll}
E\{X(t)\}=0, & \text { for all } t \in T . \\
E\left\{|X(t)|^{2}\right\}<\infty, & \text { for all } t \in T .
\end{array}
$$

The convariance function of the field $K(t, s)=E\{X(t) \cdot \overline{X(s)}\}$

is a continuous positive definite function on $T \times T$.

For each $g \in G$,

$$
K(t, s)=K(g t, g s), \quad t, s \in T .
$$

Let $A \subset T$ be a compact set invariant under every $g \in G$ and the field $\{X(t), t \in T\}$ be observable on $A$.

For a set $S \subset T$ we shall denote by $L_{2}(X(t), t \in S)$ a Hilbert space consisting of all random variables which may be represented either as a finite linear combinations

$$
U=\sum_{i=1}^{n} c_{i} \cdot X\left(t_{i}\right)
$$

for some integer $n$, points $t_{1}, t_{2}, \cdots, t_{n}$ in $S$ and complex numbers $c_{1}, c_{2}, \cdots, c_{n}$ or as a limit in quadratic mean of such finite linear combinations under the scalar product $(U, W)$ defined by $(U, W)=E\{U \cdot \bar{W}\}$.

It is well-known that for each $g \in G$ one can define a unitary operator $U(g)$ on $L_{2}(X(t), t \in T)$ such that

$$
\begin{array}{ll}
U(g) X(t)=X(g t), & g \in G, \\
U(g) \cdot U(h)=U(g h), & g, h \in G .
\end{array}
$$

Let $H_{0}$ be a subspace of $L_{2}(X(t), t \in T)$ consisting of all random variables $u$ such that $U(g) u=u$ for all $g \in G$.

Let $\left\{u_{n}, n=1,2,3, \cdots\right\}$ be a convergent sequence of random variables in $H_{0}$ such that $\lim _{n \rightarrow \infty} u_{n}=u$. Then, since $\|u-U(g) u\| \leqq\left\|u-u_{n}\right\|+\left\|U(g) u_{n}-U(g) u\right\|=2 \cdot\left\|u-u_{n}\right\|$, for all $g \in G, U(g) u=u$, for all $g \in G$. This implies that the subspace $H_{0}$ is closed.

Let us write $H_{1}=H_{0} \cap L_{2}(X(t), t \in \Lambda)$.

For any closed subspace $H$ of $L_{2}(X(t), t \in T)$ and any random variable $u$ in $L_{2}(X(t), t \in T)$, we shall denote by Proj. $(u \mid H)$ (or simply $u \mid H$ ) the projection of $u$ onto $H$.

Let $Z \in L_{2}(X(t), t \in T)$ be such that $Z \in H_{0}$ but $Z \oplus L_{2}(X(t), t \in \Lambda)$, that is, $U(g) Z$ $=Z$, for all $g \in G$ but $Z \notin L_{2}(X(t), t \in \Lambda)$.

Let $\hat{Z}=\operatorname{Proj}\left(Z \mid L_{2}(X(t), t \in A)\right)$. Then $\hat{Z}$ is the best linear estimator of $Z$ on the basis of the realization of $\{X(t), t \in A\}$ in such a sense that $E\left\{|Z-\hat{Z}|^{2}\right\} \leqq E\left\{|Z-u|^{2}\right\}$ for any $u \in L_{2}(X(t), t \in \Lambda)$.

We shall prepare the following:

Lemma 3.1. For each $g \in G, U(g) \hat{Z}=\hat{Z}$.

Proof. $Z$ is decomposed uniquely in such a way that $Z=\hat{Z}+W$ where $W$ is a 
component of $Z$ orthogonal to $L_{2}(X(t), t \in \Lambda)$. Since for all $g \in G, Z=\hat{Z}+W=U(g) \hat{Z}$ $+U(g) W$, we have $\hat{Z}-U(g) \hat{Z}=U(g) W-W$ and hence $\|\hat{Z}-U(g) \hat{Z}\|^{2}=(\hat{Z}-U(g) \hat{Z}, U(g) W$ $-W)=(\hat{Z}, U(g) W)=\left(U(g)^{-1} \cdot \hat{Z}, W\right)=0$. Thus, we have proved lemma 3.1 .

Let $C(G)$ be a set of all bounded continuous functions on $G$.

Then we have the following lemma:

Lemma 3.2. For each pair of random variables $y, u$ in $L_{2}(X(t), t \in T)$

$$
(U(g) y, u) \in C(G) .
$$

Proof. Let $u_{n}=\sum_{k=1}^{N_{n}} c_{k, n} \cdot X\left(t_{k, n}\right), n=1,2,3, \cdots$, be such that $\lim _{n \rightarrow \infty} u_{n}=u$. Then $\left(U(g) y, u_{n}\right), n=1,2,3, \cdots$, converges uniformly to $(U(g) y, u)$ since $\mid(U(g) y, u)-(U(g) y$, $\left.u_{n}\right)|=|\left(U(g) y, u-u_{n}\right) \mid \leqq\|y\| \cdot\left\|u-u_{n}\right\|$.

Hence it is sufficient for us to prove that for all $t \in T,(U(g) y, X(t)) \in C(G)$.

For each $t \in T$, we have

$$
\begin{aligned}
|(U(g) y, X(t))-(U(h) y, X(t))| \\
\quad=\left|\left(y, U\left(g^{-1}\right) X(t)-U\left(h^{-1}\right) X(t)\right)\right| \\
\quad=\left|\left(y, X\left(g^{-1} \cdot t\right)-X\left(h^{-1} \cdot t\right)\right)\right| \\
\quad \leqq \\
\quad=2 y\|\cdot\| X\left(g^{-1} \cdot t\right)-X\left(h^{-1} \cdot t\right) \| \\
\quad\|y\| \cdot\left(K(t, t)-K\left(t, g h^{-1} \cdot t\right)\right) .
\end{aligned}
$$

Since $K(t, s)$ is continuous and $g h^{-1} \cdot t \rightarrow t$ as $g \rightarrow h$, we see that $(U(g) y, X(t)) \in C(G)$ for all $t \in T$. The boundedness of $(U(g) y, u)$ is obvious since $G$ is compact.

Let $V \subset \Lambda$ be a Borel set such that $G \cdot V=\Lambda$ and $G \cdot v_{1} \cap G \cdot v_{2}=\phi$ if $v_{1} \neq v_{2}$ $\left(v_{1}, v_{2} \in V\right)$. Since $A$ is a compact metric space invariant under every $g \in G$, we can choose such a set $V$ in $A$ (lemma 2.1 in $\S 2$ ).

Now we have the following:

Theorem 3.1. For each $\varepsilon>0$, there is a random variable $Y \in L_{2}(X(t), t \in V)$ such that

$$
E\left\{|\hat{Z}-A \cdot Y|^{2}\right\}<\varepsilon,
$$

where $A=\int_{G} U(g) d g$ whose restriction to $L_{2}(X(t), t \in \Lambda)$ is a projection operator onto $H_{1}$.

Proof. From lemma 3.2, it follows that for any fixed $y \in L_{2}(X(t), t \in T), J(u)$ $=\int_{G}(U(g) y, u) d g, u \in L_{2}(X(t), t \in T)$, is a well-defined bounded linear functional on $L_{2}(X(t), t \in T)$ where by $\int \cdot d g$ we mean the integration with respect to the normalized Haar measure on $G$.

From a theorem of Ries $z$ it follows that there exists a unique random variable $y^{*}=\int_{G} U(g) d g \cdot y$ in $L_{2}(X(t), t \in T)$ such that $J(u)=\left(y^{*}, u\right)$.

For any $\varepsilon>0$, there exist $t_{1}=g_{1} v_{1}, t_{2}=g_{2} v_{2}, \cdots, t_{n}=g_{n} v_{n}\left(g_{i} \in G, v_{i} \in V\right)$ and scalars $a_{1}, a_{2}, \cdots, a_{n}$ such that

$$
E\left\{\left|\hat{Z}-\sum_{i=1}^{n} a_{i} \cdot X\left(t_{i}\right)\right|^{2}\right\}<\varepsilon .
$$

Let us put 


$$
Y=\sum_{i=1}^{n} a_{i} \cdot X\left(v_{i}\right)
$$

Then, $Y \in L_{2}(X(t), t \in V)$.

We shall now evaluate $E\left\{|\hat{Z}-A \cdot Y|^{2}\right\}$.

We have

$$
\begin{aligned}
E\left\{|\hat{Z}-A \cdot Y|^{2}\right\} & =E\left\{\left|\hat{Z}-\int_{G} U(g) d g \cdot Y\right|^{2}\right\} \\
& =E\left\{\left|\hat{Z}-\sum_{i=1}^{n} a_{i} \int_{G} U(g) \cdot X\left(v_{i}\right) d g\right|^{2}\right\} \\
& =E\left\{\left|\hat{Z}-\sum_{i=1}^{n} a_{i} \int_{G} U\left(g g_{i}\right) \cdot X\left(v_{i}\right) d g\right|^{2}\right\}
\end{aligned}
$$

(here we have used the invariant measure $d g$ ).

Since the random variable $\hat{Z}$ is invariant with respect to all operators $U(g)$, $g \in G$ and $\int_{G} d G=1$, we have

$$
\begin{aligned}
E\left\{|\hat{Z}-A \cdot Y|^{2}\right\} & =E\left\{\left.\int_{G} U(g)\left(\hat{Z}-\sum_{i=1}^{n} a_{i} U\left(g_{i}\right) X\left(v_{i}\right)\right) d g\right|^{2}\right\} \\
& \leqq E\left\{\int_{G}\left|U(g)\left(\hat{Z}-\sum_{i=1}^{n} a_{i} X\left(t_{i}\right)\right)\right|^{2} d g\right\} \\
& =\int_{G} E\left\{\left|U(g)\left(\hat{Z}-\sum_{i=1}^{n} a_{i} X\left(t_{i}\right)\right)\right|^{2}\right\} d g \\
& =\int_{G} E\left\{\left|\hat{Z}-\sum_{i=1}^{n} a_{i} \cdot X\left(t_{i}\right)\right|^{2}\right\} d g \\
& =E\left\{\left|\hat{Z}-\sum_{i=1}^{n} a_{i} X\left(t_{i}\right)\right|^{2}\right\}<\varepsilon .
\end{aligned}
$$

Now, we shall show that for any $u \in L_{2}(X(t), t \in A)$,

$$
\int_{G} U(g) d g \cdot u=\operatorname{Proj}\left(u \mid H_{1}\right) \text {. }
$$

From our assumptions, the operator $U(\cdot)$ is a continuous unitary representation of $G$ on $L_{2}(X(t), t \in T)$.

Hence, the representation $\left(U(g), L_{2}(X(t), t \in T)\right)$ is completely reducible.

It is well-known that equivalent classes of irreducible representations for a compact group is only countably many and each irreducible representation is finite dimensional.

Thus, $L_{2}(X(t), t \in T)$ is decomposed into the direct sum of closed subspaces $V_{j}$, $j=1,2,3, \cdots$ such that

$$
L_{2}(X(t), t \in T)=V_{1}+V_{2}+\cdots+V_{n}+\cdots
$$

and also for each $j(j=1,2,3, \cdots), V_{j}$ is a direct sum of $d_{j}$-dimensional ( $d_{j}$ is finite) subspaces $V_{j k}, k=1,2,3, \cdots$ such that

$$
V_{j}=V_{j_{1}}+V_{j_{2}}+\cdots+V_{j k}+\cdots
$$

where (i) the decomposition (7) is unique, (ii) for each $j(j=1,2,3, \cdots)$, the dimension of $V_{j k}, k=1,2,2, \cdots$, are identical and $d_{j}$, (iii) for each $j, k(j, k=1,2,3, \cdots), V_{j k}$ is 
an invariant subspace under the operators $U(g), g \in G$, and for each $k(k=1,2,3, \cdots)$ the restriction of $U(\cdot)$ onto $V_{j k}$ (in symbols $M^{(j)}(\cdot)=U(\cdot) \mid V_{j k}$ ) is an irreducible unitary representation of $G$ with dimension $d_{j}$ and identical for each $k(k=1,2,3, \cdots)$, (iv) $M^{\left(j_{1}\right)}(g)$ and $M^{\left(j_{2}\right)}(g)$ are inequivalent if $j_{1} \neq j_{2}$.

Let us choose an arbitrary orthonormal basis $\left(e_{j k}^{(1)}, e_{j k}^{(2)}, \cdots, e_{j k}^{\left(d j^{j}\right.}\right)$ of the subspace $V_{j k}$ and fix it. Then the operator $M^{(j)}(g)$ is written by $d_{j} \times d_{j}$ matrix

$$
M^{(j)}(g)=\left\{m_{l k}^{(j)}(g)\right\}, \quad l, k=1,2,3, \cdots, d_{j} .
$$

It is well-known that for each $l, k, j\left(l, k=1,2,3, \cdots, d_{j}, j=1,2,3, \cdots\right), m_{l k}^{(j)}(g)$ is a bounded continuous function on $G$ and hence square-integrable with respect to the invariant measure on $G$.

The following orthogonality relations are well-known:

$$
\int_{G} m_{l k}^{(j)}(g) \overline{m_{l^{\prime} k^{\prime}}^{\left(j^{\prime}\right)}(g)} d g=\frac{1}{d_{j}} \cdot \delta_{l \cdot l^{\prime}} \cdot \delta_{k \cdot k^{\prime}} \cdot \delta_{j \cdot j^{\prime}}
$$

where $\delta_{i, j}=1$ if $i=j ;=0$ otherwise.

These relations are independent of the choice of coordinate systems.

Let $M^{(1)}(g)=U(g) \mid V_{1 k}, k=1,2,3, \cdots$, be the irreducible identity representation of $G$, that is, $U(g) \mid V_{1 k}=I$. Then, from Schur's lemma the irreducible identity representation of $G$ is one-dimensional.

Hence, $d_{1}=1, M^{(1)}(g)=1$ and

$$
V_{1}=H_{0}=V_{11}+V_{12}+\cdots+V_{1 k}+\cdots,
$$

where $H_{0}=\{u \mid U(g) u=u$, for all $g \in G\} \subset L_{2}(X(t), t \in T)$.

Thus, from the orthogonality relations it follows that for each $j(j=2,3,4, \cdots)$, for all $\nu, \mu=1,2,3, \cdots, d_{j}$,

$$
\int_{G} m_{\nu \mu}^{(j)}(g) d g=0
$$

Let $y, u \in L_{2}(X(t), t \in T)$ be any elements but fixed. Let $y_{j}=\operatorname{Proj}\left(y \mid V_{j}\right)$, $u_{j}=\operatorname{Proj}\left(u \mid V_{j}\right)$ and $y_{j k}=\operatorname{Proj}\left(y \mid V_{j k}\right), u_{j k}=\operatorname{Proj}\left(u \mid V_{j k}\right)$ and

$$
\begin{aligned}
& y_{j k}=y_{j k}^{(j)} e_{j k}^{(1)}+y_{j k}^{(2)} e_{j k}^{(2)}+\cdots+y_{j k}^{(d j)} e_{j k}^{(d j)}, \\
& u_{j k}=u_{j k}^{(1)} e_{j k}^{(1)}+u_{j k}^{(2)} e_{j k}^{(2)}+\cdots+u_{j k}^{(d)} e_{j k}^{(d j)} .
\end{aligned}
$$

Then we have

$$
\begin{aligned}
\int_{G}(U(g) y, u) d g & =\sum_{j=1}^{\infty} \sum_{k=1}^{\infty} \int_{G}\left(M^{(j)}(g) y_{j k}, u_{j k}\right) d g \\
& =\sum_{j=1}^{\infty} \sum_{k=1}^{\infty} \sum_{\nu=1}^{\infty} \sum_{\mu=1}^{\infty} y_{j k}^{(\nu)} \cdot \overline{u_{j k}^{(\mu)}} \cdot \int_{G} m_{\nu \mu}^{(j)}(g) d g \\
& =\sum_{k=1}^{\infty} y_{j k}^{(1)} \cdot \overline{u_{1 k}^{(1)}}=\left(y_{1}, u_{1}\right) \\
& =\left(y_{1}, u\right) \\
& =\left(\operatorname{Proj}\left(y \mid V_{1}\right), u\right) \\
& =\left(\int_{G} U(g) d g \cdot y, u\right) .
\end{aligned}
$$


Thus, the operator $\int_{G} U(g) d g$ is a projection onto the closed subspace $H_{0}$. It is obvious that

$$
\begin{aligned}
\int_{G} U(g) d g \cdot L_{2}(X(t), t \in \Lambda) & =H_{0} \cap L_{2}(X(t), t \in \Lambda) \\
& =H_{1} .
\end{aligned}
$$

This completes the proof.

Example 3.1. Let $R_{2}$ be the 2-dimensional Euclidean space. Let each point in $R_{2}$ be written by the polar coordinate $(r, \theta), 0 \leqq r<\infty, 0 \leqq \theta \leqq 2 \pi$.

Let $G=S O(2)$ and $A$ be a compact set such that

$$
\Lambda=\left\{(r, \theta) \in R_{2} \mid 0<\rho_{1} \leqq r \leqq \rho_{2}<\infty, 0 \leqq \theta \leqq 2 \pi\right\} .
$$

Then $G \cdot A=A$. Thus, as a Borel cross section of $A$, we may choose a set $V=\{(r, 0) \mid$ $\left.\rho_{1} \leqq r \leqq \rho_{2}\right\}$, since $V$ obviously satisfies the conditions:

(i) $A=G \cdot V$,

(ii) $G v_{1} \cap G v_{2}=\phi$ if $v_{1} \neq v_{2}\left(v_{1}, v_{2} \in V\right)$.

Let $\left\{X(r, \theta),(r, \theta) \in R_{2}\right\}$ be a complex-valued homogeneous random field such that $E\{X(r, \theta)\}=0$, for all $(r, \theta) \in R_{2}, E\left\{|X(r, \theta)|^{2}\right\}<\infty$, for all $(r, \theta) \in R_{2}$, the covariance function $K\left((r, \theta),\left(r^{\prime}, \theta^{\prime}\right)\right)=E\left\{X(r, \theta) \cdot \overline{X\left(r^{\prime}, \theta^{\prime}\right)}\right\}=K\left((r, 0),\left(r^{\prime}, \theta^{\prime}-\theta\right)\right)$, for all $(r, \theta),\left(r^{\prime}, \theta^{\prime}\right)$ $\in R_{2}$ and $K\left((r, \theta),\left(r^{\prime}, \theta^{\prime}\right)\right)$ is a continuous positive definite function on $R_{2} \times R_{2}$.

For each $g \in S O(2)$ such that

$$
g=\left(\begin{array}{rr}
\cos \theta, & \sin \theta \\
-\sin \theta, & \cos \theta
\end{array}\right), \quad 0 \leqq \theta<2 \pi,
$$

we shall define a unitary operator $U(\theta), 0 \leqq \theta<2 \pi$, by writing for each $\left(r, \theta^{*}\right) \in R_{2}$,

$$
U(\theta) \cdot X\left(r, \theta^{*}\right)=X\left(r, \theta^{* *}\right), \quad 0 \leqq \theta^{* *}<2 \pi,
$$

where $\theta+\theta^{*} \equiv \theta^{* *}, \bmod 2 \pi$.

Let $Z=X(0,0)$. Then, $U(\theta) Z=Z$, for all $\theta(0 \leqq \theta \leqq 2 \pi)$.

Thus, from theorem 3.1, it follows that for each $\varepsilon>0$ there exists a random variable $Y$ such that

$$
E\left\{\left|\hat{Z}-\frac{1}{2 \pi} \int_{0}^{2 \pi} U(\theta) d \theta \cdot Y\right|^{2}\right\}<\varepsilon,
$$

where (a) $Y$ is a finite linear combination of the field on $V$ such that for some integer $N$, points $\left(r_{1}, 0\right),\left(r_{2}, 0\right), \cdots,\left(r_{N}, 0\right)$ in $V$ and complex numbers $c_{1}, c_{2}, \cdots, c_{N}$

$$
Y=\sum_{k=1}^{N} c_{k} X\left(r_{k}, 0\right),
$$

(b) $\hat{Z}$ is the best linear estimator of $Z=X(0,0)$ on the basis of the realizations of $\{X(t), t \in \Lambda\}$.

\section{$\S 4$. On the optimal linear interpolation of homogeneous random fields II.}

\subsection{Summary.}

In this section we shall continue considering a problem of interpolation for a homogeneous random field $\{X(t), t \in T\}$ on a metric space $T$ acted upon by a compact 
metric group $G$.

The field $\{X(t), t \in T\}$ is observable on a compact set $\Lambda$ invariant under every $g \in G$.

Let $Z \in L_{2}(X(t), t \in T)$ be a random variable invariant with respect to all unitary operators $U(g), g \in G$, defined on $L_{2}(X(t), t \in T)$ by writing $U(g) X(t)=X(g t), t \in T$. Let $\hat{Z} \in L_{2}(X(t), t \in A)$ be the best linear estimator of $Z$ based on realizations of $X(t)$ on $\Lambda$

We shall proceed further in details to construct a sequence of random variables $\left\{Y_{n}, n=1,2,3, \cdots\right\}$ such that

(i) $Y_{n} \in L_{2}(X(t), t \in V), n=1,2,3,4, \cdots$,

(ii) $\hat{Z}=1.1 . \mathrm{i} . \mathrm{n} \int_{G} U(g) d g \cdot Y_{n}$,

where $Y_{n}, n=1,2,3, \cdots$, are concretely calculated on the basis of the realizations of the field on a Borel cross section $V$ of $\Lambda$ by making use of the theory of the reproducing kernel Hilbert space generated by the covariance function of the field and the previous result in theorem 2.1 of $\S 2$ on decomposability of measures on a compact metric space.

Definitions and notations appeared in the previous sections are used in this section as well with the same meanings.

\subsection{An approximating sequence for the optimal linear interpolation.}

Let $T$ and $G$ be the spaces identical with those defined in $\S 3$.

For any metric space $X$, we shall denote by $\mathfrak{B}_{X}$ the $\sigma$-field of Borel subsets of $X$ and $C(X)$ a set of all bounded continuous functions on $X$.

Let $\{X(t), t \in T\}$ be a complex-valued homogeneous random field measurable with respect to $\mathfrak{B}_{T}$ satisfying the conditions:

$$
\begin{array}{ll}
E\{X(t)\}=0, & \text { for all } t \in T . \\
E\left\{|X(t)|^{2}\right\}<\infty, & \text { for all } t \in T .
\end{array}
$$

The covariance function $K(t, s)=E\{X(t) \overline{X(s)}\}$ is a continuous positive definite function on $T \times T$.

The field $\{X(t), t \in T\}$ is observable on a compact set $A \subset T$ such that for every $g \in G, g \cdot \Lambda=\Lambda$.

For a set $S \subset T, L_{2}(X(t), t \in S)$ denotes the Hilbert space same as defined in $\S 3$.

By $U(g), g \in G$ we denote unitary operators defined on $L_{2}(X(t), t \in T)$ by writing $U(g) X(t)=X(g t), t \in T$.

Let $V \subset A$ be a Borel cross section of $A$, that is, $V$ is a Borel subset of $A$ such that

(i) $G \cdot V=\Lambda$,

(ii) $G \cdot v_{1} \cap G \cdot v_{2}=\phi$ if $v_{1} \neq v_{2}\left(v_{1}, v_{2} \in V\right)$.

Suppose that for any $g \in G, g \neq e$, there is no fixed point in $\Lambda$. Then, the mapping

$$
\xi:(g, v) \rightarrow g v
$$

is a Borel isomorphism between $G \times V$ and $A$ (see lemma 2.2 in $\S 2$ ). 
Let $\mu$ be a measure on $A$ invariant under every $g \in G$ such that the support of $\mu$ is $A$. Let us denote by $\mu^{*}$ the induced measure on $G \times V$ from $\mu$ on $\Lambda$ by the Borel isomorphism $\xi$, that is, $\mu^{*}$ is a measure on $G \times V$ such that for any $A \times B \in \mathfrak{B}_{G \times V}$,

$$
\mu^{*}(A \times B)=\mu(\xi(A \times B)) .
$$

Without loss of generality, we assume that $\mu^{*}(G \times V)=1$, in other words, $\mu^{*}$ is a probability measure on $G \times V$. From theorem 2.1 in $\S 2$, it follows that $\mu^{*}$ may be decomposed into a direct product measure of the normalized Haar measure of $G$ and a probability measure $m$ on $V$ such that for any $A \times B \in \mathfrak{B}_{G \times V}$,

$$
\mu^{*}(A \times B)=\nu(A) \cdot m(B) \text {. }
$$

Thus, we may and do identify the space $A$ with the product space $G \times V$ and the measure $\mu$ on $\Lambda$ with the measure $\mu^{*}$ on $G \times V$.

Let $Z \in L_{2}(X(t), t \in T)$ be such that

(i) For all $g \in G, U(g) Z=Z$,

(ii) $Z \notin L_{2}(X(t), t \in \Lambda)$.

Let $\hat{Z}$ be the best linear estimator of $Z$ on the basis of the realizations of the field $X(t)$ on $A$. Actually, $\hat{Z}$ is the projection of $Z$ onto $L_{2}(X(t), t \in \Lambda)$, that is,

$$
\hat{Z}=\operatorname{Proj}\left(Z \mid L_{2}(X(t), t \in A)\right) .
$$

It has been shown in lemma 3.1 in $\S 3$ that for each $g \in G, U(g) \hat{Z}=\hat{Z}$.

Since the covariance function $K(t, s)$ restricted to $A \times A$ is also continuous and positive definite, it follows from a theorem of Aronszajn (see thorem 6.4 in $\S 6$ ) that $K(t, s), t, s \in \Lambda$, generates a unique reproducing kernel Hilbert space $H(K)$.

$H(K)$ is actually a Hilbert space consisting of functions on $\Lambda$ satisfying the conditions :

(K.1) For each $t \in \Lambda, K(t, \cdot) \in H(K)$.

(K.2) For any $f \in H(K),(f, K(t, \cdot))_{K}=f(t), t \in \Lambda$,

where by $\left(f_{1}, f_{2}\right)_{K}$ we denote the scalar product of every pair of elements $f_{1}, f_{2}$ in $H(K)$.

We shall make use of the following theorem:

Theorem 4.1. ([12], page 10, THEOREM 2D).

There is a one-one, scalar product preserving linear mapping $\phi$ from $H(K)$ onto $L_{2}(X(t), t \in \Lambda)$ such that

$$
\phi(K(t, \cdot))=X(t), \quad t \in \Lambda
$$

Since the mapping $\phi$ is an isometric isomorphism between $H(K)$ and $L_{2}(X(t), t \in \Lambda)$, one can define for each $g \in G$ a unitary operator $U^{*}(g)$ on $H(K)$ by writing

$$
U(g) \cdot \phi(f)=\phi\left(U^{*}(g) f\right), \quad \text { for each } f \in H(K) .
$$

The operators $\left\{U^{*}(g), g \in G\right\}$ obviously satisfy the following:

$$
U^{*}(g) \cdot U^{*}(h)=U^{*}(g h), \quad \text { for all } g, h \in G .
$$

We shall prepare the following: 
Lemma 4.1. For any $u \in L_{2}(X(t), t \in \Lambda)$,

$$
\left(\phi^{-1}(u)\right)(t)=E\{u \cdot \overline{X(t)}\}, \quad t \in \Lambda .
$$

Proof. Since $\phi$ is an isometric isomorphism between $H(K)$ and $L_{2}(X(t), t \in \Lambda)$, there always exists a unique function $h \in H(K)$ such that $\phi(h)=u$. Since $\phi$ is scalar product preserving,

$$
\begin{aligned}
E\{u \cdot \overline{X(t)}\} & =(u, X(t))=(\phi(h), \phi(K(t, \cdot))) \\
& =(h, K(t, \cdot))_{K}=h(t), \quad t \in A .
\end{aligned}
$$

This shows that $\left(\phi^{-1}(u)\right)(t)=E\{u \cdot \overline{X(t)}\}, t \in A$.

Let us denote by $\Sigma$ a set of all functions in $H(K)$ invariant under every $U^{*}(g)$, $g \in G$, that is,

$$
\Sigma=\left\{f \in H(K) \mid U^{*}(g) f=f, \text { for all } g \in G\right\} .
$$

Since $H_{1}=\left\{u \in L_{2}(X(t), t \in A) \mid U(g) u=u\right.$, for all $\left.g \in G\right\}$ is a closed subspace in $L_{2}(X(t), t \in \Lambda)$ and $\Sigma$ is an inverse image of $H_{1}$ under $\psi, \Sigma$ is a closed subspace of $H(K)$.

Now, let us define an operator $K^{*}$ on $C(V)$ in such a manner that for any $f \equiv C(V)$,

$$
\left(K^{*} f\right)(t)=\int_{V} K(v, t) f(v) d m(v), \quad t \in \Lambda .
$$

Then, we have the following:

Lemma 4.2. (i) $K^{*} \cdot C(V) \subset H(K) \subset C(\Lambda)$.

(ii) For any $f \in C(V)$,

$$
\phi\left(K^{*} f\right)=\int_{V} X(v) f(v) d m(v) .
$$

Thus, for any $f \in C(V), \phi\left(K^{*} f\right) \in L_{2}(X(t), t \in V)$.

Proof. Let us consider a random variable

$$
u=\int_{V} X(v) f(v) d m(v), \quad \text { for } f \in C(V) .
$$

Since $E\left\{|u|^{2}\right\}<\infty$, the random variable $u$ is well-defined and $u \in L_{2}(X(t), t \in V)$. From lemma 4.1, it follows that

$$
\begin{aligned}
\left(K^{*} f\right)(t) & =\int_{V} K(v, t) f(v) d m(v) \\
& =\int_{V} E\{X(v) \overline{X(t)}\} f(v) d m(v) \\
& =E\{u \cdot \overline{X(t)}\} \\
& =\left(\phi^{-1}(u)\right)(t), \quad t \in \Lambda
\end{aligned}
$$

Thus $\phi\left(K^{*} \cdot f\right)=\int_{V} X(v) f(v) d m(v)$.

Let $h \in H(K)$. Then, for any $t, s \in \Lambda$,

$$
\begin{aligned}
|h(t)-h(s)| & =\left|(h, K(t, \cdot)-K(s, \cdot))_{K}\right| \\
& \leqq\|h\|_{K} \cdot \sqrt{K(t, t)+K(s, s)-2 K(t, s)} .
\end{aligned}
$$


Thus, $h$ is continuous. Since $\Lambda$ is compact, $h$ is bounded. Thus, $h \in C(\Lambda)$. This completes the proof of lemma 4.2 .

Since for each pair of random variables $y, u$ in $L_{2}(X(t), t \in A),(U(g) y, u) \in C(G)$, (lemma 3.2 in $\S 3$ ), the operator

$$
A=\int_{G} U(g) d g
$$

is well-defined on $L_{2}(X(t), t \in A)$. $A$ is a projection operator on $L_{2}(X(t), t \in A)$ onto the closed subspace $H_{1}$ (theorem 3.1 in $\S 3$ ).

Let us define an operator $A^{*}$ on $H(K)$ in such a manner that for each $h \in H(K)$,

$$
A \cdot \phi(h)=\phi\left(A^{*} \cdot h\right) \text {. }
$$

Then, $A^{*}=\int_{G} U^{*}(g) d g$, since for any $u \in L_{2}(X(t), t \in \Lambda)$,

$$
\begin{aligned}
(A \cdot \psi(h), u) & =\int_{G}(U(g) \cdot \psi(h), u) d g \\
& =\int_{G}\left(\psi\left(U^{*}(g) h\right), u\right) d g \\
& =\int_{G}\left(U^{*}(g) h, \phi^{-1}(u)\right)_{K} d g \\
& =\left(\int_{G} U^{*}(g) d g \cdot h, \phi^{-1}(u)\right)_{K} \\
& =\left(\phi\left(\int_{G} U^{*}(g) d g \cdot h\right), u\right) \\
& =\left(\psi\left(A^{*} \cdot h\right), u\right) .
\end{aligned}
$$

This implies that $A^{*}=\int_{G} U^{*}(g) d g$.

Lemma 4.3. For any $f \in H(K)$ and any $g \in G$,

$$
\left(U^{*}(g) f\right)(t)=f\left(g^{-1} \cdot t\right), \quad t \in \Lambda .
$$

In particular, if $f \in \Sigma$, then for all $g \in G$ and $t \in A, f(g t)=f(t)$.

Proof. From (5) and (6), it follows that $U(g) X(t)=X(g t)=\psi(K(g t, \cdot))=$ $\phi\left(U^{*}(g) K(t, \cdot)\right)$. Hence, we have for any $g \in G$, and any $t, s \in \Lambda$,

Thus, it follows that for any $f \in H(K)$,

$$
\begin{aligned}
\left(U^{*}(g) K(t, \cdot)\right)(s) & =K(g t, s) \\
& =K\left(t, g^{-1} \cdot s\right) .
\end{aligned}
$$

$$
\begin{aligned}
\left(U^{*}(g) f\right)(t) & =\left(U^{*}(g) f, K(t, \cdot)\right)_{K} \\
& =\left(f, U^{*}\left(g^{-1}\right) K(t, \cdot)\right)_{K} \\
& =\left(f, K\left(g^{-1} \cdot t, \cdot\right)\right)_{K} \\
& =f\left(g^{-1} \cdot t\right), \quad t \in \Lambda .
\end{aligned}
$$

If $f \in \Sigma$, then $\left(U^{*}(g) f\right)(t)=f(t), t \in \Lambda$. Thus, $f\left(g^{-1} \cdot t\right)=f(t)$, for all $g \in G$. This is equivalent to the statement that $f(g t)=f(t)$ for all $g \in G$.

We shall define an operator $K$ on a Hilbert space $L_{2}\left(\Lambda, \mathfrak{B}_{\Lambda}, \mu\right)$ consisting of all 
square integrable functions on $\Lambda$ with respect to the measure $\mu$ in such a way that for any $f \in L_{2}\left(\Lambda, \mathfrak{B}_{A}, \mu\right)$,

$$
(K f)(t)=\int_{\Lambda} K(s, t) f(s) d \mu(s), \quad t \in \Lambda
$$

We have the following:

Lemma 4.4. For any $h \in \Sigma, A^{*} K^{*} h=K h$ and $K \cdot \Sigma \subset H(K)$.

Proof. Suppose $h \in \Sigma$, and let us consider the following random variables:

$$
\begin{aligned}
& u_{1}=\int_{V} X(v) h(v) d m(v), \\
& u_{2}=\int_{\Lambda} X(t) h(t) d \mu(t) .
\end{aligned}
$$

Then, it is obvious that $u_{1}$ and $u_{2}$ are well-defined and $u_{1} \in L_{2}(X(t), t \in V)$ and $u_{\text {. }}$ $\in L_{2}(X(t), t \in \Lambda)$. From lemma 4.2, it follows that $u_{1}=\psi\left(K^{*} \cdot h\right)$.

Now, we have

$$
\begin{aligned}
\left(\phi^{-1}\left(u_{2}\right), K(t, \cdot)\right)_{K} & =E\left\{u_{2} \overline{X(t)}\right\} \\
& =\int_{A} E\{X(s) \overline{X(t)}\} h(s) d \mu(s) \\
& =\int_{\Lambda} K(s, t) h(s) d \mu(s) \\
& =(K \cdot h)(t), \quad t \in \Lambda .
\end{aligned}
$$

This implies that $K \cdot \Sigma \subset H(K)$ and

$$
\phi(K \cdot h)=\int_{\Lambda} X(t) h(t) d \mu(t) .
$$

Thus, for any $g \in G$, any $f \in H(K)$ and any $h \in \Sigma$,

$$
\begin{aligned}
\left(f, U^{*}(g) K^{*} \cdot h\right)_{K} & =\left(\phi(f), \phi\left(U^{*}(g) K^{*} \cdot h\right)\right) \\
& =\left(\phi(f), U(g) \cdot \phi\left(K^{*} \cdot h\right)\right) \\
& =\left(\phi(f), U(g) \cdot \int_{V} X(s) h(s) d m(s)\right) \\
& =\left(\phi(f), \int_{V} X(g s) h(s) d m(s)\right) \\
& =\int_{V} E\{\psi(f) \cdot \overline{X(g s)}\} \overline{h(s)} d m(s) \\
& =\int_{V} f(g s) \overline{h(s)} d m(s) .
\end{aligned}
$$

Hence, we have

$$
\begin{aligned}
\left(f, A^{*} \cdot K^{*} \cdot h\right)_{K} & =\int_{G}\left(f, U^{*}(g) K^{*} \cdot h\right)_{K} d g \\
& =\int_{G} d g \cdot \int_{V} f(g s) \overline{h(s)} d m(s) \\
& =\int_{G} \int_{V} f(g s) \overline{h(s)} d m(s) d g .
\end{aligned}
$$


Since for any $h \in \Sigma, h(t)=h(g t), t \in \Lambda$,

$$
\begin{aligned}
\left(f, A^{*} \cdot K^{*} \cdot h\right)_{K} & =\int_{G} \int_{V} f(g s) \overline{h(g s)} d m(s) d g \\
& =\int_{\Lambda} f(t) \overline{h(t)} d \mu(t) \\
& =\int_{A} E\{\psi(f) \cdot \overline{X(t)}\} \overline{h(t)} d \mu(t) \\
& =E\left\{\phi(f) \cdot \overline{\left.\int_{\Lambda}^{X(t) h(t) d \mu(t)}\right\}}\right. \\
& =(\phi(f), \phi(K h)) \\
& =(f, K h)_{K} .
\end{aligned}
$$

Thus, for any $h \in \Sigma, A^{*} \cdot K^{*} \cdot h=K \cdot h$. The proof of lemma 4.4 is now complete.

Lemma 4.5. Let $\rho_{z}(t)=E\{Z \cdot \overline{X(t)}\}, t \in A$. Then, $\rho_{z} \in \Sigma$.

Proof. Since for any $t \in A, \rho_{z}(t)=E\{Z \cdot \overline{X(t)}\}=E\{\hat{Z} \cdot \overline{X(t)}\}, \rho_{z} \in H(K)$. Since $\hat{Z}$ is invariant with respect to all $U(g), g \in G$, we have for any $g \in G, \hat{Z}=\phi\left(\rho_{z}\right)=U(g) \hat{Z}$ $=U(g) \cdot \psi\left(\rho_{z}\right)=\psi\left(U^{*}(g) \cdot \rho_{z}\right)$. Thus, $\rho_{z}=U^{*}(g) \cdot \rho_{z}$, that is, $\rho_{z} \in \Sigma$.

Lemma 4.6.

(i) For any $H \in C(V)$,

$$
A^{*} K^{*} H-\rho_{z} \in \Sigma
$$

(ii) For any constant $\alpha$,

$$
\left(I^{*}-\alpha A^{*} K^{*}\right) \Sigma \subset \Sigma,
$$

where $I^{*}$ is the identity operator on $H(K)$.

Proof. First, we shall prove that for any $g \in G, U^{*}(g) A^{*}=A^{*}$. For any $f, h$ $\in H(K)$ and $g \in G$,

$$
\begin{aligned}
\left(f, U^{*}(g) A^{*} h\right)_{K} & =\left(U^{*}\left(g^{-1}\right) f, A^{*} h\right)_{K} \\
& =\int_{G}\left(U^{*}\left(g^{-1}\right) f, U^{*}\left(g^{*}\right) h\right)_{K} d g^{*} \\
& =\int_{G}\left(f, U^{*}(g) U^{*}\left(g^{*}\right) h\right)_{K} d g^{*} \\
& =\int_{G}\left(f, U^{*}\left(g g^{*}\right) h\right)_{K} d g^{*} \\
& =\int_{G}\left(f, U^{*}\left(g^{\prime}\right) h\right)_{K} d g^{\prime} \\
& =\left(f, A^{*} h\right)_{K} .
\end{aligned}
$$

Since $K^{*} C(V) \subset H(K), A^{*} K^{*} H$ is well-defined and an element in $H(K)$. Thus, for any $g \in G$,

$$
\begin{gathered}
U^{*}(g)\left(A^{*} K^{*} H-\rho_{z}\right)=U^{*}(g) A^{*} K^{*} H-U^{*}(g) \rho_{z} \\
=A^{*} K^{*} H-\rho_{z} .
\end{gathered}
$$

This implies that $A^{*} K^{*} H-\rho_{z} \in \Sigma$. 
Now, we shall prove the second half of lemma 4.6.

For any $h \in \Sigma$ and any $g \in G$,

$$
\begin{aligned}
U^{*}(g)\left(I^{*}-\alpha \cdot A^{*} K^{*}\right) h & =U^{*}(g) h-\alpha \cdot U^{*}(g) A^{*} K^{*} h \\
& =h-\alpha \cdot A^{*} K^{*} h \\
& =\left(I^{*}-\alpha \cdot A^{*} K^{*}\right) \cdot h .
\end{aligned}
$$

Thus, the proof of lemma 4.6 is now complete.

Here, we have the following:

Theorem 4.2. Let $\left\{H_{n}, n=1,2,3, \cdots\right\}$ be a sequence of functions in $C(V)$ defined recursively in such a way that

$$
H_{n}(t)=H_{n-1}(t)-\alpha \cdot\left(A^{*} K^{*} H_{n-1}(t)-\rho_{z}(t)\right), \quad t \in V,
$$

where $\alpha$ is a constant and $H_{0}(t)$ is an arbitrary function in $C(V)$.

Let us define a sequence of random variables $\left\{Y_{n}, n=1,2,3, \cdots\right\}$ in such a way that

$$
Y_{n}=\int_{V} X(s) H_{n}(s) d m(s), \quad n=1,2,3, \cdots .
$$

Then, (i) $Y_{n} \in L_{2}(X(t), t \in V)$, for all $n(n=1,2,3, \cdots)$.

(ii) For a constant $\alpha$ such that $0<\alpha<2 / D$, where $D=\int_{A} K(t, t) d \mu(t)$,

$$
\hat{Z}=\lim _{n \rightarrow \infty} \int_{G} U(g) d g \cdot Y_{n} .
$$

In order to prove theorem 4.2 , we shall first need to derive several lemmas.

Since $\int_{\Lambda} \int_{\Lambda}|K(t, s)|^{2} d \mu(t) d \mu(s)<\infty$, that is, the operator $K$ is compact on $L_{2}\left(\Lambda, \mathfrak{B}_{\Lambda}, \mu\right)$, the eigen-values of the operator $K$ are only countably many.

Let $\lambda_{\nu}, \nu=1,2,3, \cdots$, be non-trivial eigen-values and $\varphi_{\nu}(t), t \in \Lambda, \nu=1,2,3, \cdots$, be the corresponding normalized eigen-functions of the covariance function $K(t, s)$ such that

$$
\lambda_{\nu} \cdot \varphi_{\nu}(t)=\int_{\Lambda} K(s, t) \cdot \varphi_{\nu}(s) d \mu(s), \quad t \in \Lambda .
$$

Since $K(t, s), t, s \in \Lambda$, is positive definite, the eigen-values $\lambda_{\nu}, \nu=1,2,3, \cdots$, are positive real numbers.

It is well-known that the covariance function $K(t, s)$ may be expanded as follows:

$$
K(t, s)=\sum_{\nu=1}^{\infty} \lambda_{\nu} \cdot \overline{\varphi_{\nu}(t)} \cdot \varphi_{\nu}(s), \quad t, s \in \Lambda .
$$

(Cf. [14], page 278, Mercer's theorem).

Lemma 4.7. The family of all eigen-functions $\left\{\varphi_{\nu}, \nu=1,2,3, \cdots\right\}$ spans the Hilbert space $L_{2}\left(\Lambda, \mathfrak{B}_{\Lambda}, \mu\right)$.

Proof. Let $f \in L_{2}\left(\Lambda, \mathfrak{B}_{\Lambda}, \mu\right)$ be such that for all $\nu=1,2,3, \cdots$,

$$
\left(f, \varphi_{\nu}\right)_{\Lambda}=\int_{\Lambda} f(t) \cdot \overline{\varphi_{\nu}(t)} d \mu(t)=0,
$$

where we denote by $(f, h)_{\Lambda}$ the scalar product of each pair of functions $f, h$ in $L_{2}\left(\Lambda, \mathfrak{B}_{\Lambda}, \mu\right)$. 
Then, from (8), it follows that

$$
\begin{array}{r}
\int_{A} \int_{A} K(t, s) f(t) \overline{f(s)} d \mu(t) d \mu(s) \\
=\sum_{\nu=1}^{\infty} \lambda_{\nu} \cdot\left|\left(f, \varphi_{\nu}\right)_{\Lambda}\right|^{2}=0 .
\end{array}
$$

Since $K(t, s)$ is positive definite, $f$ must be a zero element in $L_{2}\left(A, \mathfrak{B}_{A}, \mu\right)$. This implies that $L_{2}\left(A, \mathfrak{B}_{\Lambda}, \mu\right)$ is spanned by $\left\{\varphi_{\nu}, \nu=1,2,3, \cdots\right\}$.

Lemma 4.8.

(i) For each $\nu(\nu=1,2,3, \cdots), \varphi_{\nu} \in H(K)$.

(ii) $\left(\varphi_{,}, \varphi_{\mu}\right)_{K}=\lambda_{\nu}^{-1}$, if $\nu=\mu$,

$$
=0, \quad \text { if } \quad \nu \neq \mu \text {. }
$$

(iii) $\phi\left(\varphi_{2}\right)=\lambda_{\nu}^{-1} \cdot \int_{\Lambda} X(s) \cdot \varphi_{\nu}(s) d \mu(s)$.

Proof. Let us write

$$
X_{k}=\int_{\Lambda} X(s) \cdot \varphi_{k}(s) d \mu(s), \quad k=1,2,3, \cdots .
$$

It is easily seen that $E\left\{\left|X_{k}\right|^{2}\right\}=\lambda_{k}<\infty$ and $X_{k} \in L_{2}(X(t), t \in A)$. Since $\left(\psi^{-1}\left(X_{k}\right)\right)(t)$ $=E\left\{X_{k} \cdot \overline{X(t)}\right\}=\lambda_{k} \cdot \varphi_{k}(t), t \in \Lambda$,

$$
X_{k}=\phi\left(\lambda_{k} \cdot \varphi_{k}\right)=\lambda_{k} \cdot \phi\left(\varphi_{k}\right) .
$$

Thus, $\varphi_{k} \subseteq H(K)$ and $\phi\left(\varphi_{k}\right)=\lambda_{k}^{-1} \cdot X_{k}$.

Since $E\left\{X_{k} \cdot \bar{X}_{n}\right\}=\lambda_{k}$ if $k=n ;=0$ if $k \neq n$,

$$
\begin{aligned}
\left(\varphi_{k}, \varphi_{n}\right)_{K} & =\left(\phi\left(\varphi_{k}\right), \phi\left(\varphi_{n}\right)\right) \\
& =\lambda_{k}^{-1} \cdot \lambda_{n}^{-1} \cdot E\left\{X_{k} \cdot \bar{X}_{n}\right\} \\
& =\lambda_{k}^{-1}, \quad \text { if } \quad k=n, \\
& =0, \quad \text { if } \quad k \neq n .
\end{aligned}
$$

Thus, we have proved lemma 4.8.

Lemma 4.9. For any $h \in H(K)$,

$$
\|h\|_{K}^{2}=\sum_{\nu=1}^{\infty} \lambda_{\nu}^{-1} \cdot\left|\left(h, \varphi_{\nu}\right)_{A}\right|^{2}<\infty .
$$

Proof. Since $H(K) \subset L_{2}\left(A, \mathfrak{B}_{\mathcal{A}}, \mu\right), h$ may be expanded as follows :

$$
h(t)=\sum_{\nu=1}^{\infty}\left(h, \varphi_{\nu}\right)_{\Lambda} \cdot \varphi_{\nu}(t), \quad t \in A .
$$

Thus, we have

$$
\begin{aligned}
\|h\|_{K}^{2} & =\sum_{\nu=1}^{\infty} \sum_{\mu=1}^{\infty}\left(h, \varphi_{\nu}\right)_{A} \cdot \overline{\left(h, \varphi_{\mu}\right)_{A}} \cdot\left(\varphi_{\nu}, \varphi_{\mu}\right)_{K} \\
& =\sum_{\nu=1}^{\infty} \lambda_{\nu}^{-1} \cdot\left|\left(h, \varphi_{\nu}\right)_{A}\right|^{2} .
\end{aligned}
$$

This completes the proof of lemma 4.9.

Lemma 4.10. Let $B(\alpha)$ be such that 


$$
B(\alpha)=\sup _{\substack{\|\|_{K}=1 \\ f \in \Sigma}}\left\|\left(I^{*}-\alpha \cdot A^{*} \cdot K^{*}\right) f\right\|_{K}
$$

where $\alpha$ is a constant.

Then, $B(\alpha)<1$ if $0<\alpha<2 / D$, where $D=\int_{A} K(t, t) d \mu(t)$.

Proof. For any $h \in \Sigma$, we have the following expansion:

$$
h(t)=\sum_{\nu=1}^{\infty}\left(h, \varphi_{\nu}\right)_{\Lambda} \cdot \varphi_{\nu}(t), \quad t \in \Lambda .
$$

Thus, from lemma 4.4, it follows that

Hence, we have

$$
A^{*} \cdot K^{*} \cdot h=K \cdot h=\sum_{\nu=1}^{\infty} \lambda_{\nu} \cdot\left(h, \varphi_{\nu}\right)_{A} \cdot \varphi_{\nu} .
$$

$$
\left(I^{*}-\alpha \cdot A^{*} \cdot K^{*}\right) h=\sum_{\nu=1}^{\infty}\left(1-\alpha \cdot \lambda_{\nu}\right)\left(h, \varphi_{\nu}\right)_{\Lambda} \cdot \varphi_{\nu} .
$$

Thus, we have the following inequality:

$$
\begin{aligned}
\left\|\left(I^{*}-\alpha \cdot A^{*} \cdot K^{*}\right) h\right\|_{K}^{2} & =\sum_{\nu=1}^{\infty} \lambda_{\nu}^{-1} \cdot\left|1-\alpha \cdot \lambda_{\nu}\right|^{2} \cdot\left|\left(h, \varphi_{\nu}\right)_{A}\right|^{2} \\
& <\sum_{\nu=1}^{\infty} \lambda_{\nu}^{-1} \cdot\left|\left(h, \varphi_{\nu}\right)_{\Lambda}\right|^{2}=\|h\|_{K}^{2},
\end{aligned}
$$

if for all $\nu=1,2,3, \cdots,\left|1-\alpha \cdot \lambda_{2}\right|<1$.

This condition can be satisfied by choosing the constant $\alpha$ such that $2 / \lambda^{*}>\alpha>0$, where $\lambda^{*}=\operatorname{Max}\left(\lambda_{1}, \lambda_{2}, \lambda_{3}, \cdots, \lambda_{n}, \cdots\right)$.

Since $D=\int_{A} K(t, t) d \mu(t)=\sum_{\nu=1}^{\infty} \lambda_{\nu}>\lambda^{*}$, if $\alpha$ is such that $0<\alpha<2 / D$,

$$
\left\|\left(I^{*}-\alpha \cdot A^{*} \cdot K^{*}\right) h\right\|_{K}^{2}<\|h\|_{K}^{2}, \quad \text { for any } h \in \Sigma .
$$

Thus, we have proved lemma 4.10 .

We are now ready to derive the proof of theorem 4.2 .

Let us apply the operator $A^{*} \cdot K^{*}$ on both sides of (7). Then, we have

$$
A^{*} \cdot K^{*} \cdot H_{n}=A^{*} \cdot K^{*} \cdot H_{n-1}-\alpha \cdot A^{*} \cdot K^{*}\left(A^{*} \cdot K^{*} \cdot H_{n-1}-\rho_{z}\right) .
$$

Thus, for each $n(n=1,2,3, \cdots)$,

$$
\begin{aligned}
A^{*} \cdot K^{*} \cdot H_{n}-\rho_{z} & =\left(I^{*}-\alpha \cdot A^{*} \cdot K^{*}\right)\left(A^{*} \cdot K^{*} \cdot H_{n-1}-\rho_{z}\right) \\
& =\left(I^{*}-\alpha \cdot A^{*} \cdot K^{*}\right)^{n} \cdot\left(A^{*} \cdot K^{*} \cdot H_{0}-\rho_{z}\right) .
\end{aligned}
$$

Since $A^{*} \cdot K^{*} \cdot H_{0}-\rho_{z} \in \Sigma$, we readily find the following inequality:

$$
\begin{aligned}
\left\|A^{*} \cdot K^{*} \cdot H_{n}-\rho_{z}\right\|_{K} & =\left\|\left(I^{*}-\alpha \cdot A^{*} \cdot K^{*}\right)^{n} \cdot\left(A^{*} \cdot K^{*} \cdot H_{0}-\rho_{z}\right)\right\|_{K} \\
& \leqq B(\alpha)^{n} \cdot\left\|A^{*} \cdot K^{*} \cdot H_{0}-\rho_{z}\right\|_{K} .
\end{aligned}
$$

Hence, by choosing the constant $\alpha$ such that $B(\alpha)<1$, we have

$$
\left\|A^{*} \cdot K^{*} \cdot H_{n}-\rho_{z}\right\|_{K} \rightarrow 0 \text {, as } n \rightarrow \infty \text {. }
$$

From lemma 4.2 , it follows that for each $n(n=1,2,3, \cdots)$, 


$$
Y_{n}=\int_{V} X(s) H_{n}(s) d m(s)
$$

is well-defined and $Y_{n} \in L_{2}(X(t), t \in V)$.

Since $Y_{n}=\psi\left(K^{*} \cdot H_{n}\right), \quad A \cdot Y_{n}=\phi\left(A^{*} \cdot K^{*} \cdot H_{n}\right)$, where $A=\int_{G^{*}} U(g) d g$ and $A^{*}$ $=\int_{G} U *(g) d g$.

Thus, it follows that

$$
\begin{aligned}
\left\|A^{*} \cdot K^{*} \cdot H_{n}-\rho_{z}\right\|_{K} & =\left\|\phi\left(A^{*} \cdot K^{*} \cdot H_{n}\right)-\phi\left(\rho_{z}\right)\right\| \\
& =\left\|A \cdot Y_{n}-\hat{Z}\right\| \rightarrow 0 \text { as } n \rightarrow \infty .
\end{aligned}
$$

This completes the proof of theorem 4.2.

Example 4.1. Let $T$ be the unit sphere with radius $c$ in $R_{3}$, that is, $T=\{(x, y, z)$ $\left.\in R_{3} \mid x^{2}+y^{2}+z^{2}=c^{2}\right\}$.

Let $(\theta, \varphi), 0 \leqq \theta \leqq \pi, 0 \leqq \varphi<2 \pi$, be such that

where $(x, y, z) \in T$.

$$
\begin{aligned}
& x=c \cdot \sin \theta \cdot \cos \varphi, \\
& y=c \cdot \sin \theta \cdot \sin \varphi, \\
& z=c \cdot \cos \theta,
\end{aligned}
$$

Let $G$ be a subgroup of $S O(3)$ such that each element of $G$ is of the form:

$$
g=\left(\begin{array}{rrr}
\cos \eta, & \sin \eta, & 0 \\
-\sin \eta, & \cos \eta, & 0 \\
0, & 0, & 1
\end{array}\right), \quad 0 \leqq \eta \leqq 2 \pi,
$$

that is, $G$ is a group consisting of all rotations around the $z$-axis.

Let $\{X(\theta, \varphi),(\theta, \varphi) \in T\}$ be a homogeneous random field on $T$ such that

(i) $E\{X(\theta, \varphi)\}=0$, for all $(\theta, \varphi) \in T$,

(ii) $E\left\{|X(\theta, \varphi)|^{2}\right\}<\infty$, for all $(\theta, \varphi) \in T$,

(iii) $K\left((\theta, \varphi),\left(\theta^{\prime}, \varphi^{\prime}\right)\right)=E\left\{X(\theta, \varphi) \cdot \overline{X\left(\theta^{\prime}, \varphi^{\prime}\right)}\right\}$

$$
=K\left((\theta, 0),\left(\theta^{\prime}, \varphi^{\prime}-\varphi\right)\right) \text {, for all }(\theta, \varphi),\left(\theta^{\prime}, \varphi^{\prime}\right) \in T \text {. }
$$

Let us define unitary operators $U(\varphi), 0 \leqq \varphi<2 \pi$, on $L_{2}(X(t), t \in T)$ by writing

$$
U(\varphi), X\left(\theta, \varphi^{\prime}\right)=X\left(\theta, \varphi^{\prime \prime}\right),
$$

where $0 \leqq \varphi^{\prime \prime}<2 \pi, \varphi+\varphi^{\prime} \equiv \varphi^{\prime \prime}, \bmod 2 \pi$.

Let $A$ be such that

$$
\Lambda=\left\{(\theta, \varphi) \mid 0<\rho_{1} \leqq \theta \leqq \rho_{2} \leqq \pi, 0 \leqq \varphi<2 \pi\right\} .
$$

Then, we may choose as a Borel cross section of $\Lambda$ a set $V$ such that

$$
V=\left\{(\theta, 0) \mid \rho_{1} \leqq \theta \leqq \rho_{2}\right\} .
$$

Suppose that the field $\{X(\theta, \varphi),(\theta, \varphi) \in T\}$ is observable on $A$.

Let $\mu$ be a probability measure distributing uniformly on $T$. Then, it is written as follows: 


$$
d \mu(\theta, \varphi)=\frac{1}{2} \sin \theta d \theta \cdot \frac{1}{2 \pi} d \varphi .
$$

Let $Z=X(0,0)$. Then, $Z$ is invariant with respect to every $U(\varphi), 0 \leqq \varphi<2 \pi$.

Now, let a sequence of functions $\left\{H_{n}, n=1,2,3, \cdots\right\}$ be defined recursively as follows :

$$
H_{n}(t)=H_{n-1}(t)-\alpha \cdot\left(A^{*} \cdot \int_{0}^{\pi} K((\theta, 0), t) H_{n-1}(\theta, 0) d m(\theta)-\rho_{z}(t)\right)
$$

where $\rho_{z}(t)=E\{X(0,0) \cdot \overline{X(t)}\}, t \in A$,

$$
\begin{aligned}
& d m(\theta)=\frac{1}{2}-\sin \theta d \theta, \\
& H_{0}(t) \equiv 1, \quad t \in \Lambda .
\end{aligned}
$$

Then, from theorem 4.2, it follows that

$$
\hat{Z}=\lim _{n \rightarrow \infty} \int_{0}^{2 \pi} U(\varphi) d \varphi \cdot Y_{n}
$$

where $Y_{n}=\int_{0}^{\pi} X(\theta, 0) H_{n}(\theta, 0) d m(\theta)$.

\section{§5. On the uniformly minimum variance unbiased linear estimates of mean value functions of homogeneous random fields.}

\subsection{Summary.}

In this section we shall consider an estimation problem of mean value functions $m(t)$ of homogeneous random fields $\{X(t), t \in T\}$ on a compact metric space $T$ acted upon by a compact metric group $G$.

We assume that mean value functions $m(t)$ are $G$-invariant, that is, $m(t)=m(g t)$, for all $g \in G$ and $t \in T$.

Let $V$ be a Borel cross section of $T$, in other words, $V$ be a subset of $T$ such that $G \cdot V=T$ and $G \cdot v_{1} \cap G \cdot v_{2}=\phi$ if $v_{1} \neq v_{2}\left(v_{1}, v_{2} \in V\right)$.

Then, it is shown that the uniformly minimum variance unbiased linear estimate $\widehat{m(t)}$ of the mean value function $m(t)$ admits the expression

$$
\widehat{m(t)}=\int_{G} U(g) d g \cdot X(v), \quad v \in V,
$$

where $t=g^{\prime} \cdot v$ for some $g^{\prime} \in G$ and $v \in V$, and $\{U(g), g \in G\}$ are unitary operators on $L_{2}(X(t), t \in T)$ defined by writing $U(g) X(t)=X(g t)$.

\subsection{An estimation problem of mean value functions.}

Let $T$ be a compact metric space acted upon by a compact metric group $G$ such that (i) $G \cdot T=T$, (ii) each $g \in G$ is a homeomorphism acting on $T$ and (iii) the mapping $(g, t) \rightarrow g t$ from $G \times T$ onto $T$ is continuous.

From our assumptions, it follows that there exists a Borel subset $V$ of $T$ such that $G \cdot V=T$ and $G \cdot v_{1} \cap G \cdot v_{2}=\phi$ if $v_{1} \neq v_{2}\left(v_{1}, v_{2} \in V\right)$, (see lemma 2.1 in $\S 2$ ).

Let $\{X(t), t \in T\}$ be a homogeneous random field on $T$ with the following properties :

(1) $E\left\{|X(t)|^{2}\right\}<\infty$, for all $t \in T$. 
(2) The covariance function $K(t, s)=\operatorname{Cov}(X(t), X(s))$ is continuous positive definite function on $T \times T$.

(3) For each $g \in G, K(t, s)=K(g t, g s), t, s \in T$.

Let us denote the mean value function of the field by

$$
m(t)=E\{X(t)\}, \quad t \in T .
$$

Let $L_{2}(X(t), t \in T)$ be the Hilbert space consisting of all random variables which may be represented either as a finite linear combination

$$
u=\sum_{i=1}^{n} c_{i} \cdot X\left(t_{i}\right)
$$

for some integer $n$, points $t_{1}, t_{2}, \cdots, t_{n}$ in $T$ and scalars $c_{1}, c_{2}, \cdots, c_{n}$ or as a limit in quadratic mean of such finite linear combinations under the scalar product $(u, z)$ defined by

$$
(u, z)=E_{m}\{u \cdot \bar{z}\}=\operatorname{Cov}(u, z)+E_{m}\{u\} \cdot E_{m}\{\bar{z}\} .
$$

The subscript $m$ on an expectation operator $E$ is written to indicate that the expectation is computed under the assumption that $m(\cdot)$ is the true mean value function.

Since the covariance function $K(t, s), t, s \in T$, is continuous positive definite, it follows from a theorem of Aronszajn (see theorem 6.4 in $\S 6$ ) that $K(t, s)$ generates a unique reproducing kernel Hilbert space $H(K)$.

$H(K)$ is actually a Hilbert space consisting of functions on $T$ satisfying the conditions :

(K.1) For each $t \in T, K(t, \cdot) \in H(K)$.

(K.2) For any $f \in H(K)$,

$$
(f, K(t, \cdot))_{K}=f(t), \quad t \in T,
$$

where by $(f, h)_{K}$ we denote the scalar product of each pair of functions $f, h$ in $H(K)$.

Let $M$ be an arbitrarily given subset of $H(K)$ and let us assume that $m \in M$.

We shall make use of the following theorem:

Theorem 5.1. ([11], page 29, Theorem 4A). There is a linear one-one mapping $\phi$ from $H(K)$ onto $L_{2}(X(t), t \in T)$ with the following properties:

(L.1) For each $t \in T, \phi(K(t, \cdot))=X(t)$.

(L.2) For any $f \in H(K)$,

$$
E_{m}\{\phi(f)\}=(f, m)_{K}, \quad \text { for all } m \in M .
$$

(L.3) For any $f, h \in H(K)$,

$$
\operatorname{Cov}(\phi(f), \phi(h))=(f, h)_{K} .
$$

We shall make use of the definition of an unbiased linear estimate of the mean value function $m(t), t \in T$, introduced by E. Parzen [11].

A random variable $\phi(h), h \in H(K)$, is said to be an unbiased linear estimate of the value $m(t)$ at a particular point $t \in T$ of the mean value function $m(\cdot)$ if

$$
E_{m}\{\phi(h)\}=(h, m)_{K}=m(t), \quad \text { for all } m \in M .
$$

For any Hilbert space $H$ and its closed subspace $H^{*}$ and any element $u \in H$, we shall denote by 
the projection of $u$ onto $H^{*}$.

$$
\operatorname{Proj}\left(u \mid H^{*}\right)
$$

We shall use the following:

Theorem 5.2. ([11], page 29, theorem $4 \mathrm{~A}$ ). The uniformly minimum variance unbiased linear estimate $\widehat{m(t)}$ of $m(t) \in M$ is given by

$$
\widehat{m(t)}=\phi(\operatorname{Proj}(K(t, \cdot) \mid \bar{M}))
$$

where $\bar{M}$ is the smallest closed subspace of $H(K)$ containing $M$.

Now, let us write

$$
\Sigma=\{f \in H(K) \mid f(t)=f(g t), \text { for all } g \in G\} .
$$

Hereafter, we shall assume that the mean value function $m(t)$ is invariant under every $g \in G$, in other words,

$$
m(\cdot) \in \Sigma \text {. }
$$

For each $g \in G$, let us define a linear operator $U(g)$ on $L_{2}(X(t), t \in T)$ by writing $U(g) X(t)=X(g t), t \in T$. Then, since $m \in \Sigma$ and

$$
\begin{aligned}
(U(g) X(t), U(g) X(s)) & =\operatorname{Cov}(X(g t), X(g s))+m(g t) \overline{m(g s)} \\
& =K(t, s)+m(t) \overline{m(s)} \\
& =(X(t), X(s)),
\end{aligned}
$$

the operators $U(g), g \in G$, are unitary.

Since the following inequality holds:

$$
\begin{aligned}
\|f-h\|_{K}^{2} & \leqq\|\phi(f)-\phi(h)\|^{2} \\
& \leqq\left(1+\|m\|_{K}^{2}\right) \cdot\|f-h\|_{K}^{2}, \quad \text { for any } f, h \in H(K),
\end{aligned}
$$

the mapping $\phi$ is a linear homeomorphism between $H(K)$ and $L_{2}(X(t), t \in T)$.

Lemma 5.1. Let $B$ be a closed linear subspace of $H(K)$ and the mean value function $m \in B$. Then, for any $f \in H(K)$,

$$
\phi(\operatorname{Proj}(f \mid B))=\operatorname{Proj}(\phi(f) \mid \psi(B)) .
$$

Proof. Since $\phi$ is a homeomorphism between $H(K)$ and $L_{2}(X(t), t \in T), \phi(B)$ is closed.

For any $f \in H(K)$, there is a unique decomposition such that

$$
f=f_{1}+f_{2}
$$

where $f_{1}=\operatorname{Proj}(f \mid B)$ and $f_{2}=\operatorname{Proj}\left(f \mid B^{\perp}\right)$.

Let $u_{1}=\operatorname{Proj}(\phi(f) \mid \phi(B))$ and $u_{2}=\operatorname{Proj}\left(\phi(f) \mid \psi(B)^{\perp}\right)$. Then, $\phi(f)=u_{1}+u_{2}=\phi\left(f_{1}\right)$ $+\phi\left(f_{2}\right)$. have

Since $u_{1} \in \phi(B)$, there is a unique function $k \in B$ such that $\phi(k)=u_{1}$. Thus, we

$$
\begin{aligned}
\left\|\psi\left(f_{1}\right)-u_{1}\right\|^{2} & =\left(u_{1}, \phi\left(f_{2}\right)\right)-\left(\psi\left(f_{1}\right), \phi\left(f_{2}\right)\right) \\
& =(m, k)_{K}\left(m, f_{2}\right)_{K}-\left(m, f_{1}\right)_{K}\left(m, f_{2}\right)_{K} \\
& =0
\end{aligned}
$$


since $m \in B$ and $f_{2} \in B^{\perp}$.

Lemma 5.2. Let $V_{1}=\{u \mid U(g) u=u$, for all $g \in G\} \subset L_{2}(X(t), t \in T)$. Then, $V_{1}$ is a closed subspace of $L_{2}(X(t), t \in T)$ and $\phi(\Sigma)=V_{1}$. Thus, $\Sigma$ is a closed subspace of $H(K)$.

Proof. For any $u_{1}, u_{2} \in V_{1}$ and any scalars $\alpha, \beta$,

$$
\begin{aligned}
U(g)\left(\alpha u_{1}+\beta u_{2}\right) & =\alpha \cdot U(g) u_{1}+\beta U(g) u_{2} \\
& =\alpha u_{1}+\beta u_{2} .
\end{aligned}
$$

Let $\left\{u_{n}, n=1,2,3, \cdots\right\}$ be a sequence in $V_{1}$ such that for $u^{*} \in L_{2}(X(t), t \in T)$,

Then, for any $g \in G$,

$$
\left\|u_{n}-u^{*}\right\| \rightarrow 0 \text { as } n \rightarrow \infty \text {. }
$$

$$
\begin{aligned}
\left\|u^{*}-U(g) u^{*}\right\| & \leqq\left\|u^{*}-u_{n}\right\|+\left\|U(g) u_{n}-U(g) u^{*}\right\| \\
& =2 \cdot\left\|u_{n}-u^{*}\right\| \rightarrow 0, \text { as } n \rightarrow \infty .
\end{aligned}
$$

Thus, $u^{*} \in V_{1}$ and hence $V_{1}$ is closed.

For any $h \in \Sigma$ and any $g \in G$,

$$
\begin{aligned}
(U(g) \psi(h), X(t)) & =\left(\psi(h), U\left(g^{-1}\right) X(t)\right) \\
& =\left(\psi(h), X\left(g^{-1} \cdot t\right)\right) \\
& =\left(h, K\left(g^{-1} \cdot t, \cdot\right)\right)_{K}+(h, m)_{K}\left(m, K\left(g^{-1} t, \cdot\right)\right)_{K} \\
& =h\left(g^{-1} t\right)+(h, m)_{K} m\left(g^{-1} t\right) \\
& =h(t)+(h, m)_{K} m(t) \\
& =(\psi(h), X(t)), \quad \text { for all } \quad t \in T .
\end{aligned}
$$

This implies that $U(g) \psi(h)=\psi(h)$, for all $g \in G$. Thus, $\phi(\Sigma) \subset V_{1}$.

Conversely, for any $z \in V_{1}$, there exists a unique function $f \in H(K)$ such that $\phi(f)=z$.

Since $U(g) \psi(f)=\psi(f)$, for all $g \in G$, we have

$$
\begin{aligned}
(\psi(f), X(t)) & =f(t)+(f, m)_{K} m(t) \\
& =(U(g) \phi(f), X(t)) \\
& =\left(\phi(f), X\left(g^{-1} t\right)\right) \\
& =f\left(g^{-1} t\right)+(f, m)_{K} \cdot m(t), \quad \text { for all } t \in T .
\end{aligned}
$$

Thus, it follows that $f(t)=f\left(g^{-1} t\right)$, for all $g \in G$ and $t \in T$.

This implies that $f \in \Sigma$. Thus, we have proved that

$$
V_{1}=\phi(\Sigma)
$$

Since $V_{1}$ is closed and $\phi$ is a homeomorphism, $\Sigma$ is closed.

We have now the following:

Theorem 5.3. Let $\widehat{m(t)}$ be the uniformly minimum variance unbiased linear estimate of the value $m(t)$ at the point $t \in T$ of the mean value function $m \in \Sigma$.

Then, $\widehat{m(t)}$ is written as follows: 


$$
\widehat{m(t)}=\int_{G} U(g) d g \cdot X(v),
$$

where $t=g^{*} v$ for some $g^{*} \in G$ and $v \in V$.

If $T$ is a homogeneous space, that is, $G$ is transitive on $T$, then the Borel cross section $V$ consisting of a single point $v_{0} \in T$ and

$$
\widehat{m(t)}=\int_{G} U(g) d g \cdot X\left(v_{0}\right) \text {. }
$$

Proof. First, we shall show that the operator $\int_{G} U(g) d g$ is well-defined.

Since for any fixed $y \in L_{2}(X(t), t \in T)$ and for all $t \in T$,

$$
\begin{aligned}
& |(U(g) y, X(t))-(U(h) y, X(t))| \\
& \quad \leqq 2 \cdot\|y\| \cdot\left(K(t, t)-K\left(t, g h^{-1} t\right)\right), \quad g, h \in G,
\end{aligned}
$$

$(U(g) y, z) \in C(G)$, for any fixed $z \in L_{2}(X(t), t \in T)$, where $C(G)$ is a set of all bounded continuous functions on $G$. Hence, it is clear that the operator $\int_{G} U(g) d g$ is welldefined on $L_{2}(X(t), t \in T)$.

By the same arguments in the proof of theorem 3.1 in $\S 3$, we see that the operator $\int_{G} U(g) d g$ is the projection operator onto the closed subspace $V_{1}$ of $L_{2}(X(t)$, $t \in T$ ).

From theorem 5.2 and lemma 5.1, it follows that the uniformly minimum variance unbiased linear estimate $\widehat{m(t)}$ of $m(t), t=g * v \in T(g * \in G, v \in V)$, is given by

$$
\begin{aligned}
\widehat{m(t)} & =\phi(\operatorname{Proj}(K(t, \cdot) \mid \Sigma)) \\
& =\operatorname{Proj}(\psi(K(t, \cdot)) \mid \psi(\Sigma)) \\
& =\operatorname{Proj}\left(X(t) \mid V_{1}\right) \\
& =\int_{G} U(g) d g \cdot X(t) .
\end{aligned}
$$

Since $X(t)=X(g * v)=U(g *) X(v)$, it is written as follows :

$$
\begin{aligned}
\widehat{m(t)} & =\int_{G} U(g) d g \cdot U\left(g^{*}\right) X(v) \\
& =\int_{G} U(g) U\left(g^{*}\right) d g \cdot X(v) \\
& =\int_{G} U\left(g g^{*}\right) d g \cdot X(v) \\
& =\int_{G} U(g) d g \cdot X(v),
\end{aligned}
$$

(here we have used the invariant measure $d g$ ).

If $G$ is transitive on $T$, then for any $t \in T$ and any $v_{0} \in T$, there exists $g * \in G$ such that $t=g^{*} v_{0}$.

Thus, we have 


$$
\begin{aligned}
\widehat{m(t)} & =\int_{G} U(g) d g \cdot U\left(g^{*}\right) X\left(v_{0}\right) \\
& =\int_{G} U\left(g g^{*}\right) d g \cdot X\left(v_{0}\right) \\
& =\int_{G} U(g) d g \cdot X\left(v_{0}\right) .
\end{aligned}
$$

Now, the proof of theorem 5.3 is complete.

\section{§6. Appendix.}

Theorem 6.1. ([10], page 23, theorem 4.2)

Let $X$ and $Y$ be compact metric spaces and $g$ a continuous map of $X$ onto $Y$. Then there is a Borel set $B \subset X$ such that $g(B)=Y$ and $g$ is one-one on $B$.

Theorem 6.2. ([10], page 21, theorem 3.9)

Let $X_{1}, X_{2}$ be complete separable metric spaces and $E_{1} \subset X_{1}, E_{2} \subset X_{2}$ two sets, $E_{1}$ being a Borel set. Let $\varphi$ be a measurable one-one map of $E_{1}$ into $X_{2}$ such that $\varphi\left(E_{1}\right)$ $=E_{2}$. Then $E_{2}$ is a Borel set.

Theorem 6.3. ([17], page 131)

A one-to-one continuous mapping of a compact space onto a Hausdorff space is a homeomorphism.

Theorem 6.4. ([12], page 7 , theorem $2 \mathrm{~B}$ )

A symmetric non-negative kernel $K$ generates a unique Hilbert space, which we denote by $H(K)$, of which $K$ is the reproducing kernel.

\section{§ 7. Acknowledgment.}

The author would like to express his heartiest thanks to Professor K. R. Parthasarathy of University of Manchester and Professor J. Gani of Sheffield University for their kind suggestions and encouragement while this paper was being prepared.

\section{References}

[1] Aronszajin, N.: Theory of reproducing kernels, Trans. Amer. Math. Soc., 68 (1950), $337-404$.

[2] Federer, H. ANd A.P. Morse: Some properties of measurable functions, Bull. Amer. Math. Soc., 49 (1943), 270-277.

[3] Halmos, P.: “Introduction to Hilbert Space," Chelsea, N. Y., 1951.

[4] Halmos, P. R.: “Measure Theory," Van Nostrand, Princeton, New Jersey, 1962.

[5] Hannan, E. J.: “Time Series Analysis," Methuen, London, 1960.

[6] Haninan, E. J.: Group representations and applied probability, J. Appl. Prob., 2 (1965), $1-68$.

[7] Kelley, J.L.: “General Topology," Van Nostrand, N. Y., 1961.

[8] Loeve, M.: "Probability Theory," Van Nostrand, N.Y., 1955.

[9] NAGAI, T.: On decomposability of probability measures on a separable metric space acted upon by a compact metric group, (to be published).

[10- Parthasarathy, K. R.: “Probability measures on Metric Spaces, Academic Press, N. Y., 
1967.

[11] PARZEN, E.: A new approach to the synthesis of optimal smoothing and prediction systems, Tech. Report, No. 34, Stanford Univ. 1960.

[12] PARzen, E.: Regression analysis of continuous parameter time series, II, Tech. Report, No. 35, Stanford Univ., 1960.

[13] PARzen, E.: An approach to time series analysis, Ann. Math. Statist., 32 (1961), 951-989.

[14] PARzen, E.: “Time series analysis papers," Holden-Day, Calif., 1967.

[15] Pontrjagin, L. S.: “Topological Groups," Princeton Univ. Press, Princeton, New Jersey, 1946 (Translated from Russian).

[16] Rosenblatt, M.: "Random Processes," Oxford Univ. Press, N. Y., 1962.

[17] Simmons, G. F.: “Introduction to Topology and Modern Analysis," McGraw-Hill, N. Y., 1963.

[18] Yaglom, A. M.: Second order homogeneous random fields, Fourth Berkeley Symposium on Math. Statist. and Probability, 2 (1961), 593-622. 